\title{
Vertical GaN Schottky Diodes Grown on Highly Conductive Ammono-GaN Substrate
}

\author{
P. Kruszewski ${ }^{a, b, *}$, P. Prystawko ${ }^{a, b}$, M. Grabowski ${ }^{a}$, T. Sochacki ${ }^{a}$, A. Sidor $^{a}$, \\ M. Bockowski ${ }^{a, b}$, J. Jasinski ${ }^{c}$, L. LukAsiak ${ }^{c}$, R. Kisiel ${ }^{c}$ And M. Leszczynski ${ }^{a, b}$ \\ ${ }^{a}$ Institute of High Pressure Physics, Polish Academy of Sciences, Sokolowska 29/37, 01-142 Warsaw, Poland \\ ${ }^{b}$ Top-GaN Ltd, Sokolowska 29/37, 01-142 Warsaw, Poland \\ ${ }^{c}$ Institute of Microelectronics and Optoelectronics, Warsaw University of Technology, \\ Koszykowa 75, 00-662 Warsaw, Poland
}

\begin{abstract}
We report on vertical $n$-GaN high voltage Schottky diodes grown by metal organic chemical vapour deposition and hydride vapour phase epitaxy on conductive ammono-GaN substrate. The thermionic emission current model has been applied for diodes analysis and parameters extraction. Finally, we demonstrate that breakdown voltage
\end{abstract} as high as $670 \mathrm{~V}$ for such structures can be achieved.

DOI: 10.12693/APhysPolA.134.969

PACS/topics: 85.30.Hi, 85.30.Kk, 52.59.Mv

\section{Introduction}

Wide bandgap semiconductors have become one of the most investigated materials for applications in high power, high voltage, and high frequency electronic devices operated in elevated temperatures. Thanks to high critical electric field $\left(E_{C}\right)$, wide energy bandgap $\left(E_{g}\right)$ and high value of electron saturation velocity $\left(v_{\text {sat }}\right)$, gallium nitride $(\mathrm{GaN})$ is one of the most promising candidate in this field [1-4]. However, most of fabricated GaN-based electronic devices such as diodes or field effect transistor (FET) [5] suffered from lateral geometry which limited the maximum current capability in the device. The reason of that was a lack of native and highly conductive GaN substrates. Since few years, highly conductive, high quality, and low dislocation density GaN substrates are commercially available $[6,7]$ and thus GaN-based vertical devices such as diodes or $p-n$ junctions can be fabricated.

The ideal high voltage Schottky diode (SBD) properties includes: high breakdown voltage (large critical breakdown field), low reverse current, low on-resistance, and short recovery switching time (on/off). In order to achieve these features, some fundamental obstacles should be solved first. The most critical part of a Schottky diode is high structural quality of the drift region, doping, its purity and thus electron mobility. The second thing is access to highly doped bulk substrate. This problem has been already solved and such substrates became commercially available [6-7]. Last but not least is proper design and processing of the Schottky diodes with a special care for stable and low resistance ohmic contacts to N-face GaN which still remains a big challenge [8-10].

*corresponding author; e-mail: kruszew@unipress.waw.pl
In this paper, we report on successful growth and fabrication of vertical high voltage Schottky diodes grown on truly bulk and conductive ammono-GaN substrate. In our studies, we focus mostly on breakdown voltage and the Schottky barrier height (SBH). It is worth to mention that breakdown voltages reported here are probably record values ever obtained using bulk ammono-GaN substrate.

\section{Experimental details and sample description}

In this study, we analyze two Schottky diode samples: (i) grown by metal organic chemical vapour deposition (MOCVD) and (ii) hydride vapour phase epitaxy (HVPE) growth techniques. For both samples, $n$-GaN drift layer of $1-3 \times 10^{16} \mathrm{~cm}^{-3}$ has been deposited on highly conductive, $350 \mu \mathrm{m}$ thick, $n+$ ammono-GaN substrate $\left(n \approx 1 \times 10^{19} \mathrm{~cm}^{-3}\right)$ with low threading dislocation density $\left(\approx 5 \times 10^{4} \mathrm{~cm}^{-2}\right)$. The drift region thickness were $2 \mu \mathrm{m}$ and $150 \mu \mathrm{m}$ for MOCVD and HVPE sample, respectively. The large difference in $n$-GaN thickness results from the specificity of growth techniques, in particular the growth rate which is thousand times larger for HVPE than for MOCVD. For both types of samples, circular Schottky diodes with a diameter of $60 \mu \mathrm{m}$ and standard $\mathrm{Ni} / \mathrm{Au}(200 / 1000 \mathrm{~A})$ contact metallization were therein fabricated on Ga-face of the samples. Commonly used metal stack, consisting of $\mathrm{Ti} / \mathrm{Al} / \mathrm{Ni} / \mathrm{Au}$ $(300 / 600 / 400 / 750 \mathrm{~A})$, was used for ohmic contacts deposition on the back side (N-face) of the samples. Electron beam evaporator without an in situ cleaning option (Denton Vacuum Explorer 14) was used for both ohmic and the Schottky contacts deposition. Post deposition annealing in nitrogen atmosphere at $850{ }^{\circ} \mathrm{C}$ for $1 \mathrm{~min}$ was applied for ohmic contacts formation. Both types of structures were cleaned by a standard procedure, which includes cleaning in piranha solution, acqua regia boiling, and rinsing in $\mathrm{HCl}$. Prior to metal stack deposition, 
samples have been rinsed in a solution of hydrochloride acid and $\mathrm{H}_{2} \mathrm{O}(1: 2)$. The diodes surface was not passivated. More details on the sample preparation can be find elsewhere [11].

\section{Results and discussion}

Prior to electrical characterization, samples morphology was verified using an optical microscope with Nomarski phase contrast (NDIC, Nikon LV150) and an atomic force microscopy (AFM, Veeco Dimension 3100). The images of HVPE and MOCVD $n$-GaN drift layer surface measured by AFM are presented in Fig. 1.

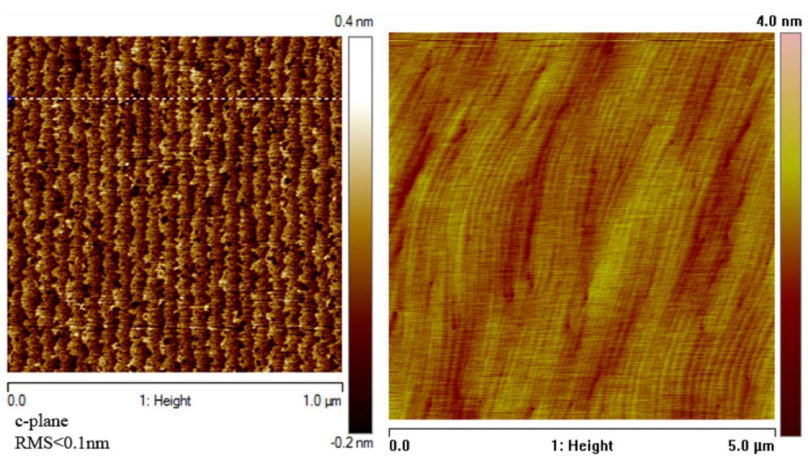

Fig. 1. AFM scans of $n$-GaN drift layer surface for HVPE and MOCVD sample, respectively.

As one can see, the surface is atomically flat with the straight atomic steps - better seen for HVPE. The surface root mean square (rms) roughness measured for a square of $1 \times 1 \mu \mathrm{m}^{2}$ is less than $0.1 \mathrm{~nm}$ in case of HVPE sample and less than $0.2 \mathrm{~nm}$ in case of MOVPE for a square $5 \times 5 \mu \mathrm{m}^{2}$. Moreover, we checked much larger areas $\left(100 \times 100 \mu \mathrm{m}^{2}\right.$ scans $)$ in several places of the samples and we have not found any significant disturbance of the atomic steps. These results confirm superior surface morphology of GaN layers grown on high quality GaN substrate.

Next, capacitance-voltage characteristics for both samples were measured at room temperature. The AC superimposed frequency was chosen to $f=1 \mathrm{MHz}$. The results of the $C-V$ measurements are depicted in Fig. 2 . As one can see, the HVPE as well as MOCVD sample, has a flat electron concentration profile in the whole measured range. For HVPE sample, $n=2.2 \times 10^{16} \mathrm{~cm}^{-3}$ while for the MOCVD, electron concentration is little bit lower and is equal to $1.3 \times 10^{16} \mathrm{~cm}^{-3}$. It is clear from Fig. 2 that our samples are highly uniform and thus we assumed that electron concentration is the same in the whole volume.

To estimate the breakdown voltage, the currentvoltage $(I-V)$ characteristics were measured for the reverse bias applied to SBD diodes and are shown in Fig. 3. Two black curves represent $I-V$ with best and typically observed breakdown voltages for HVPE sample while red curve represents $I-V$ measured for the best MOCVD diode.

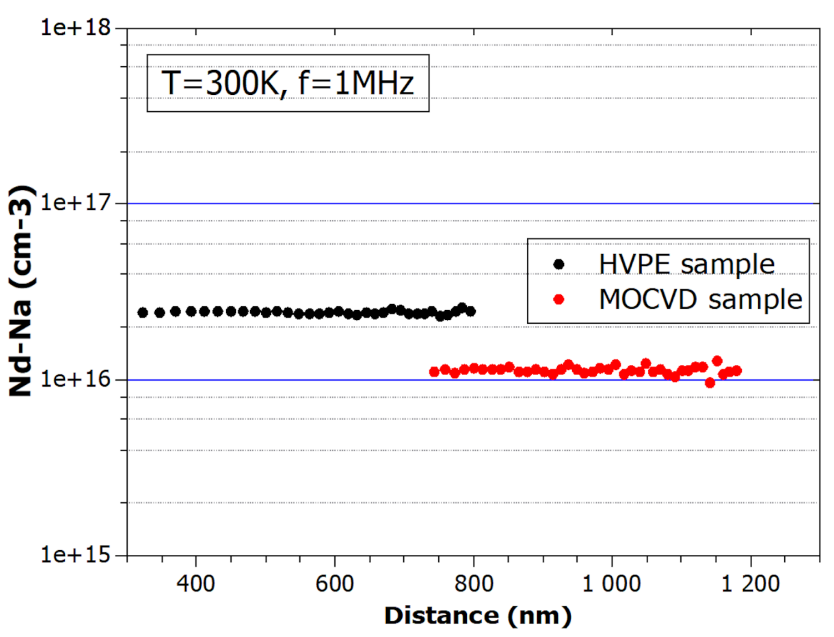

Fig. 2. Electron profile of $n$-GaN drift layer for both samples obtained from $C-V$ measurements taken at $300 \mathrm{~K}$ for a frequency of $1 \mathrm{MHz}$.

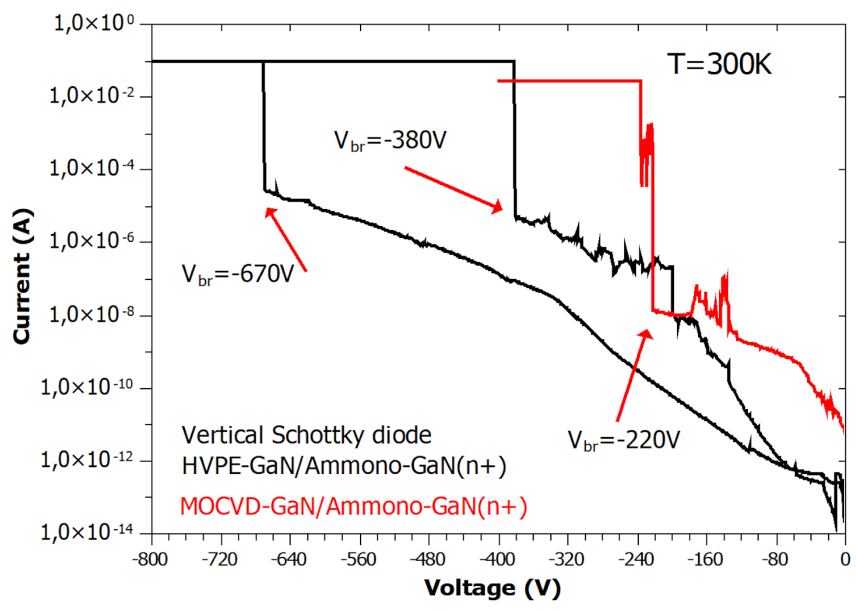

Fig. 3. Current-voltage characteristics in reverse bias regime measured for both types of the Schottky diodes at $T=300 \mathrm{~K}$..

As one can see from Fig. 3, record breakdown voltage for HVPE Schottky diode can be almost three times larger than for MOCVD diode. The MOCVD diode with largest breakdown voltage $(220 \mathrm{~V})$ is still far away when compared to typically measured HVPE diode. Moreover, the leakage current for best HVPE diode is almost two orders of magnitude lower than for MOCVD diode in whole measured voltage range.

The large difference in measured breakdown voltages for both samples arises mostly from two factors: first, HVPE sample has a drift region which is much thicker $(150 \mu \mathrm{m})$ than in MOCVD sample $(2 \mu \mathrm{m})$. This is due to the fact that GaN growth rate is much higher in HVPE than in MOCVD, and it is probably impossible to grow a thin, $2 \mu \mathrm{m}$, GaN film by HVPE technique. Additionally, as it is known from theory [1], breakdown voltage is pro- 
portional to the drift region thickness and inversely proportional to the doping. Second, in general, HVPE technique offers much more pure $n$-GaN layers and thus leads to much higher electron mobility in such films [12]. Especially, $n$-GaN layers grown by HVPE are free of carbon that creates deep acceptor states in MOCVD $n$-GaN [13].

The breakdown behaviour measured for both samples is determined by a rather complex mechanism with two dominant factors: soft breakdown with a monotonic rise of current and sudden avalanche breakdown when the electric field strength is high enough. In the case of HVPE diodes, avalanche breakdown occurs for relatively high voltages and high leakage current. For MOCVD diodes, avalanche breakdown starts even at leakage current as low as $10^{-8} \mathrm{~A}$, which is a dramatic difference comparing to HVPE diodes.

One should realize that according to theory, $2 \mu \mathrm{m}$ thick $n$-GaN drift region doped with Si: $1.3 \times 10^{16} \mathrm{~cm}^{-3}$ should make it possible to achieve breakdown voltage as high as $500 \mathrm{~V}$ [14]. In this study we obtained only half of the expected value.

Despite this fact, the breakdown voltages obtained in this study are probably record values ever reported for HVPE and MOCVD $n$-GaN layers grown on ammonoGaN bulk substrate. At the moment we are improving growth technology and device processing with special focus on edge contact termination protecting our devices from the effect of premature breakdown. We believe that these actions let us achieve even higher breakdown voltages and push our devices closer to the values predicted theoretically.

Figure 4 shows $I-V$ characteristics registered for both GaN Schottky diodes in forward bias regime. In this figure, black points represent $I-V$ curve for MOCVD diode while red ones correspond to the HVPE diode curve. The results clearly show that the MOCVD curve is shifted towards lower voltages and approximately hundred times higher current is measured for this diode than for HVPE. In both samples, series resistance limits the current flow at voltages above $0.55 \mathrm{~V}$ and this effect is much stronger for HVPE sample. One should remember that the series resistance of a Schottky diode is the sum of the resistance due to the epilayer $\left(R_{\text {epi }}\right)$ and the resistance due to the substrate $\left(R_{\text {sub }}\right)$ which is proportional to the substrate resistivity. Since both our samples were grown on conductive ammono-GaN substrate, $R_{\text {sub }}$ should be comparable in both. On the other hand, the resistance of the epi is linearly proportional to the drift region thickness. HVPE drift region is nearly a hundred times thicker than that of the MOCVD sample and thus series resistance is much higher for this sample $\left(R_{s}=440 \Omega\right)$, which is well visible in Fig. 4. The series resistance of the MOCVD sample $\left(R_{s}=12 \Omega\right)$ can be partially explained by the fact that the processed Schottky contacts were thin $(\mathrm{Ni} / \mathrm{Au} 200 \mathrm{~nm})$, which limited the maximum current flow through the structure. The contacts as thick as $1500 \mathrm{~nm}$ should be enough to sustain the current at least an order of magnitude higher.

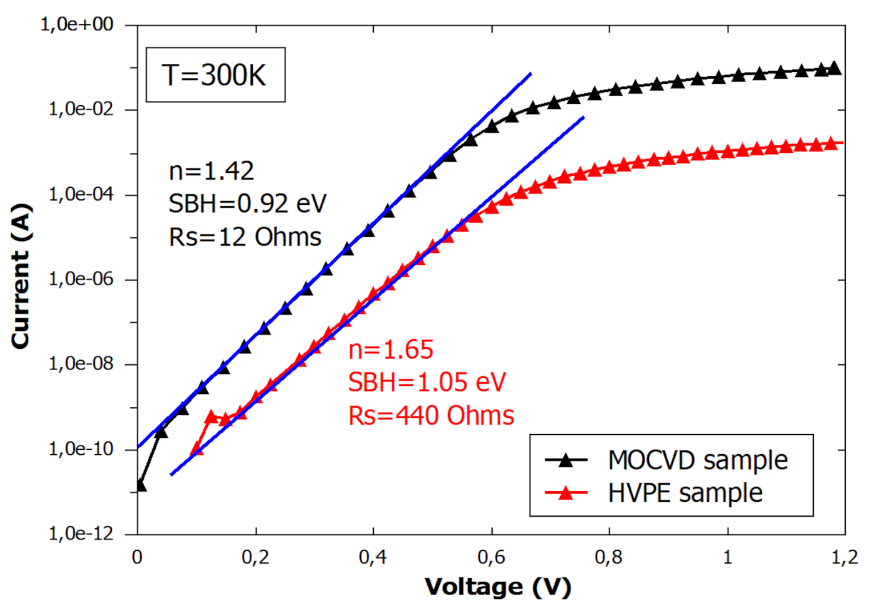

Fig. 4. The forward bias current-voltage characteristics for both types of the Schottky diodes measured at $T=300 \mathrm{~K}$.

It is well known that electron transport from metal to semiconductor can be described by simple thermionic emission theory (TE). Thereby, the Schottky barrier height and ideality factor $(n)$ can be easily estimated [11]. In this theory the forward current as a function of voltage is given by

$$
I=I_{S} \exp \left(\frac{q V_{d}}{n k T}\right)\left(1-\exp \left(\frac{-q V_{d}}{k T}\right)\right),
$$

where $V_{d}$ is the voltage across the diode, $n$ is the ideality factor and $I_{S}$ is the saturation current. Saturation current can be derived from the straight part of $I-V$ characteristic as a cross point with $y$-axis at a zero bias. Saturation current is also given by the formula

$$
I_{S}=A A^{*} T^{2} \exp \left(\frac{-q \Phi_{b}}{k T}\right),
$$

where $A^{*}$ is the effective Richardson constant (ca. $26 \mathrm{~A} \mathrm{~m}^{-2} \mathrm{~K}^{-2}$ for $\left.\mathrm{GaN}\right), A$ is the contact area, $k$ is the Boltzmann constant, $T$ is the absolute temperature, $\Phi_{b}$ is the barrier height from the metal to the semiconductor at zero bias. SBH and the ideality factor can be written respectively as

$$
\begin{aligned}
& \Phi_{b}=\frac{k T}{q} \ln \left(\frac{A A^{*} T^{2}}{I_{S}}\right), \\
& n=\frac{q}{k T}\left(\frac{\mathrm{d} V}{\mathrm{~d} \ln I}\right) .
\end{aligned}
$$

It is worth mentioning that the ideality factor is a measure of the deviation of practical diodes from the ideal thermionic emission model and can be extracted from the slope of the linear region of the $\ln I-V$ plot.

Experimentally measured $I-V$ curves have been fitted according to the proposed thermionic emission model. The results of parameters fitting lead to $S B H=0.92 \mathrm{eV}$ and $n=1.42$ and $\mathrm{SBH}=1.05$ and $n=1.65$ for MOCVD and HVPE sample, respectively. The SBH obtained within these studies are high, expectable, and 
satisfactory from the point of view of high breakdown voltage. Unfortunately, ideality factors indicate that some parallel conduction mechanisms exist in the diodes. This is probably due to generation-recombination processes and deep levels presence in the space charge region. Thus, additional defect studies i.e. deep level transient spectroscopy-based (DLTS-based) are needed to verify this.

\section{Summary}

In our work high voltage Schottky diodes grown by MOCVD and HVPE methods on conductive ammonoGaN substrate have been analyzed. We compared and discussed the results of breakdown voltage, the Schottky barrier height and ideality factor extracted from commonly used thermionic emission approach. Finally, we observed record breakdown voltage, as high as $670 \mathrm{~V}$ for HVPE sample while "only" $250 \mathrm{~V}$ for MOCVD sample. Moreover, our results suggests that breakdown voltage in GaN diodes is a two-step mechanism.

\section{Acknowledgments}

This work was financially supported by the SchottGaN Project under the Applied Research Programme of the National Centre for Research and Development, contract no PBS3/B3/29/2015 and ETNA project — "Energy Efficiency Through Novel AlGaN/GaN Heterostrucctures" within the cooperation agreement between Consiglio Nazionale delle Ricerche in Italy and Polish Academy of Sciences in Poland.

\section{References}

[1] B.J. Baliga, IEEE Electron Device Lett. 10, 455 (1989).

[2] T. Uesugi, T. Kachi, Proc. SPIE 8625, 8625V (2013).

[3] I.C. Kizilyalli, A.P. Edwards, O. Aktas, T. Prunty, D. Bour, IEEE Trans. Electron Dev. 62414 (2015).

[4] S. Chowdhury, B.L. Swenson, M.H. Wong, U.K. Mishra, Semicond. Sci. Technol. 28, 074014 (2013).

[5] S. Chowdhury, U.K. Mishra, IEEE Trans. Electron Dev. 60, 3060 (2013).

[7] Saint-Global Ceramic Materials, Lumilog.

[8] J.S. Kwak, K.Y. Lee, J.Y. Han, J. Cho, S. Chae, O.H. Nam, Y. Park, Appl. Phys. Lett. 79, 3254 (2001).

[9] T. Jang, S.N. Lee, O.H. Nam, Y. Park, Appl. Phys. Lett. 88, 193505 (2006).

[10] J.-W. Jeon, T.-Y. Seong, H. Kim, K.-K. Kim, Appl. Phys. Lett. 94, 042102 (2009).

[11] P. Kruszewski, M. Grabowski, P. Prystawko, A. Nowakowska-Siwinska, M. Sarzynski, M. Leszczynski, Phys. Status Solidi A 214, 1600376 (2017).

[12] M. Bockowski, M. Iwinska, M. Amilusik, M. Fijalkowski, B. Lucznik, T. Sochacki, Semicond. Sci. Technol. 31, 093002 (2016).

[13] Y. Cao, R. Chu, R. Li, M. Chen, R. Chang, B. Hughes, Appl. Phys. Lett. 108, 062103 (2016).

[14] Kwang H. Baik, Ph.D. Thesis, University of Florida, 2004 . 